\title{
COMPLETELY BOUNDED MAPS BETWEEN THE PREDUALS OF VON NEUMANN ALGEBRAS
}

\author{
HIROYUKI OSAKA
}

(Communicated by John B. Conway)

\begin{abstract}
Let $M$ and $N$ be von Neumann algebras. Then $B\left[M_{*}, N_{*}\right]=$ $C B\left[M_{*}, N_{*}\right]$ if and only if either $M$ is strictly finite of type I or $N$ is finitedimensional.
\end{abstract}

\section{INTRODUCTION}

In the recent development of the theory of operator algebras, it has been recognized that completely bounded maps play an important role in the study of the matricial structure of $C^{*}$-algebras (cf. [2], [12], [14]). It is of interest to know the difference between complete boundedness and mere boundedness. Smith showed in [9] that every bounded map from a $C^{*}$-algebra $A$ to a $C^{*}$-algebra $B$ is completely bounded if and only if either $A$ is finite-dimensional or $B$ is subhomogeneous. Huruya and Tomiyama showed the same result in a slightly different formulation in [3]. Recently, Effros and Ruan have systematically investigated matricially normed spaces and shown that any bounded linear map from the dual of a $C^{*}$-algebra $A$ into a $C^{*}$-algebra $B$ is completely bounded [1]. On the other hand, Ruan has shown that the only completely bounded map from a $C^{*}$-algebra $A$ into the dual of a $C^{*}$-algebra $B$ is the zero map [8]. Compared with the case of completely positive maps [10], these are contrasting results.

In this paper we shall investigate the relationship of the set of bounded maps to the subspace of completely bounded maps between the preduals of von Neumann algebras.

\section{Preliminaries}

We denote by $M_{n}$ the $n \times n$ matrix algebra over the complex number field $\mathbb{C}$. Let $A$ be a $C^{*}$-algebra and $M$ a von Neumann algebra. Let $M_{n}(A)$ be the $C^{*}$-algebra of all $n \times n$ matrices $a=\left[a_{i, j}\right]$ with entries in $A$. The $n \times n$

Received by the editors April 2, 1989.

1980 Mathematics Subject Classification (1985 Revision). Primary 46L10.

Key words and phrases. Von Neumann algebras, completely bounded maps, completely positive maps, strictly finite of type I. 
matrix space over the dual space $A^{*}, M_{n}\left(A^{*}\right)=\left\{f=\left[f_{i, j}\right] ; f_{i, j} \in A^{*}\right\}$ is regarded as the dual of $M_{n}(A)$ by

$$
\left\langle\left[a_{i, j}\right],\left[f_{i, j}\right]\right\rangle=\sum_{i, j=1}^{n} f_{i, j}\left(a_{i, j}\right),
$$

where $\left[a_{i, j}\right] \in M_{n}(A)$ and $\left[f_{i, j}\right] \in M_{n}\left(A^{*}\right)$. Let $M_{*}$ be the predual of $M$.

Let $E$ and $F$ be $C^{*}$-algebras, the duals of $C^{*}$-algebras, or the preduals of von Neumann algebras. For a linear map $\varphi: E \rightarrow F$, we define $\varphi \otimes$ $\operatorname{id}_{n}: M_{n}(E) \rightarrow M_{n}(F)$ by $\left(\varphi \otimes \mathrm{id}_{n}\right)\left(\left[a_{i, j}\right]\right)=\left[\varphi\left(a_{i, j}\right)\right]$. If $\sup _{n}\left\|\varphi \otimes \mathrm{id}_{n}\right\|<\infty$, we say that $\varphi$ is completely bounded. If $\varphi$ is completely bounded, we put the norm $\|\varphi\|_{c b}=\sup _{n}\left\|\varphi \otimes \mathrm{id}_{n}\right\|$. We denote by $B[E, F]$ and $C B[E, F]$ the set of bounded linear maps and the subspace of completely bounded maps of $E$ to $F$, respectively.

\section{MAIN RESUlt}

Let $M$ and $N$ be von Neumann algebras. The following lemma is a slight modification of [3, Lemma 1].

Lemma 2.1. Let $M$ be a von Neumann algebra containing a sequence $\left\{a_{i}\right\}_{i=1}^{\infty}$ of positive elements with $\left\|a_{i}\right\|=1$ and $a_{i} a_{j}=a_{j} a_{i}=0 \quad(i \neq j)$. Let $B$ and $C$ denote von Neumann subalgebras generated by $\left\{a_{i}\right\}_{i=1}^{n}$ and $\left\{a_{i}\right\}_{i=n+1}^{\infty}$, respectively. Then, for the integer $m=2^{n}$, there exist an element $b_{n} \in B \otimes M_{n}$ and a $\sigma$-weakly continuous linear map $\Phi_{n}: M \rightarrow M_{m}$ such that $\left\|b_{n}\right\| \leq 1$, $\left\|\Phi_{n}\right\| \leq n^{-1 / 8}, \Phi_{n} \mid C \equiv 0$, and $\left\|\left(\Phi_{n} \otimes \mathrm{id}_{m}\right)\left(b_{n}\right)\right\| \geq 2^{-3 / 2} n^{3 / 8}$.

Proof. At first we choose elements $c_{1}, \ldots, c_{n}$ of $M_{m}$ such that $\left\|c_{i}\right\|=1$ and $\left\|\sum_{i=1}^{n} \alpha_{i} c_{i}\right\| \leq\left(2 \sum_{i=1}^{n}\left|\alpha_{i}\right|^{2}\right)^{1 / 2}$ for any $\alpha_{i}$ in $\mathbb{C}, 1 \leq i \leq n$ [6], [16]. We define the $\operatorname{map} \varphi_{n}: \ell^{\infty}(n) \rightarrow M_{m}$ by

$$
\varphi_{n}(x)=\sum_{i=1}^{n} x(i) /\left(2 n^{5 / 4}\right)^{1 / 2} c_{i}, \quad x \in \ell^{\infty}(n),
$$

where $x(i)$ means the $i$ th component of $x$. By the above inequality, we have

$$
\left\|\varphi_{n}(x)\right\| \leq\left(2 \sum_{i=1}^{n}|x(i)|^{2} /\left(2 n^{5 / 4}\right)\right)^{1 / 2} \leq n^{-1 / 8}\|x\|,
$$

so that $\left\|\varphi_{n}\right\| \leq n^{-1 / 8}$.

Let $d_{n}=(1 / 2) \sum_{i=1}^{n} \delta_{i} \otimes c_{i}$, where $\delta_{i}$ is the element of $\ell^{\infty}(n)$ such that $\delta_{i}(k)=\delta_{i, k}$. Then $\left\|d_{n}\right\|=1 / 2$ and

$$
\left(\varphi_{n} \otimes \mathrm{id}_{m}\right)\left(d_{n}\right)=2^{-3 / 2} n^{-5 / 8} \sum_{i=1}^{n} c_{i} \otimes c_{i} .
$$

Here we make use of the unit vector $\mathbf{z}$ in $\mathbb{C}^{m} \otimes \mathbb{C}^{m}$ such that $\left(c_{i} \otimes c_{i}\right)(\mathbf{z})=\mathbf{z}$ for all $i$ constructed in Loebl [6]. Hence we have

$$
\left\|\varphi_{n}\right\|_{c b} \geq\left\|\left(\varphi_{n} \otimes \mathrm{id}_{m}\right)\left(d_{n}\right)\right\| \geq 2^{-3 / 2} n^{3 / 8}
$$


Suppose that $M$ acts on Hilbert space $\mathscr{H}$. Then $s\left(a_{i}\right) s\left(a_{j}\right)=s\left(a_{j}\right) s\left(a_{i}\right)=0$ $(i \neq j)$, where $s\left(a_{i}\right)$ is the support projection of $a_{i}$ in the von Neumann algebra generated by $\left\{a_{i}\right\}_{i=1}^{\infty}$. Since $a_{i} \geq 0$ and $\left\|a_{i}\right\|=1$ are assured, there exists a unit vector $\xi_{i} \in s\left(a_{i}\right) \mathscr{H}$ such that $1 / 2<\omega_{\xi_{i}}\left(a_{i}\right) \leq 1$ and $\omega_{\xi_{i}}\left(a_{j}\right)=0 \quad(i \neq j)$, where $\omega_{\xi_{i}}\left(a_{i}\right)=\left(a_{i} \xi_{i} \mid \xi_{i}\right)$. If $\omega_{\xi_{i}}\left(a_{i}\right) \neq 1$, we put $\eta_{i}=\xi_{i} /\left(\omega_{\xi_{i}}\left(a_{i}\right)\right)^{1 / 2}$; then $\omega_{\eta_{i}}\left(a_{i}\right)=1$, and $1 \leq\left\|\omega_{\eta_{i}}\right\|<2$. Hence there exists a family of normal linear functionals $\left\{\rho_{i}\right\}_{i=1}^{n}$ on $M$ such that $\rho_{i} \geq 0,\left\|\rho_{i}\right\| \leq 2$, and $\rho_{i}\left(a_{j}\right)=\delta_{i, j}$ $(1 \leq i \leq n$ and $1 \leq j<\infty)$.

We now define a completely positive linear map $\varphi: \ell^{\infty}(n) \rightarrow M$ and a $\sigma$-weakly continuous linear map $\psi: M \rightarrow \ell^{\infty}(n)$ by

$$
\varphi\left(\alpha_{1}, \ldots, \alpha_{n}\right)=\sum_{i=1}^{n} \alpha_{i} a_{i} \text { and } \psi(a)=\frac{1}{2}\left(\rho_{1}(a), \ldots, \rho_{n}(a)\right) .
$$

Both maps are contractive and, $2 \psi \varphi$ is the identity map on $\ell^{\infty}(n)$.

Put $\Phi_{n}=\varphi_{n} \psi$ and $b_{n}=\left(\varphi \otimes \mathrm{id}_{m}\right)\left(2 d_{n}\right) \in B \otimes M_{m}$. Then we have $\left\|b_{n}\right\| \leq 1$, $\left\|\Phi_{n}\right\| \leq\left\|\varphi_{n}\right\|\|\psi\| \leq n^{-1 / 8}, \Phi_{n} \mid C \equiv 0$, and

$$
\begin{aligned}
2^{-3 / 2} n^{3 / 8} & \leq\left\|\left(\varphi_{n} \otimes \mathrm{id}_{m}\right)\left(d_{n}\right)\right\| \\
& =\left\|\left(\varphi_{n}(2 \psi \varphi) \otimes \mathrm{id}_{m}\right)\left(d_{n}\right)\right\| \\
& =\left\|\left(\varphi_{n} \psi \otimes \mathrm{id}_{m}\right)\left(\varphi \otimes \mathrm{id}_{m}\right)\left(2 d_{n}\right)\right\| \\
& =\left\|\left(\Phi_{n} \otimes \mathrm{id}_{m}\right)\left(b_{n}\right)\right\| . \quad \square
\end{aligned}
$$

Definition 2.2. A finite type I von Neumann algebra $N$ is called strictly finite if $N=\bigoplus_{i=1}^{n} N_{i}$, where $N_{i}$ is of type $\mathrm{I}_{n(i)}$ and $\sup _{i} n(i)<\infty$.

Remark 2.3. From the above definition, if $N$ is not a strictly finite von Neumann algebra of type I, then we can embed $\bigoplus_{n=1}^{\infty} M_{2^{n}}$ into $N$ as a von Neumann subalgebra (cf. [13]).

Lemma 2.4. Suppose that $M$ is not a strictly finite von Neumann algebra of type I and $N$ is an infinite-dimensional von Neumann algebra. Then there exists a $\sigma$ weakly continuous bounded linear map $\Phi$ of $N$ into $M$ which is not completely bounded.

Proof. Let $a$ be a self-adjoint element of $N$ with infinite spectrum [7] and let $W^{*}(a)$ be the von Neumann subalgebra generated by $a$. By an elementary spectral argument, we can find a commuting sequence $\left\{a_{i, j}\right\}_{i, j=1}^{\infty}$ of positive element of $W^{*}(a)$ with disjoint supports and $\left\|a_{i, j}\right\|=1$. For each $n \in \mathbb{N}$, let $A_{n}$ be the von Neumann subalgebra generated by $\left\{a_{n, i}\right\}_{i=1}^{n}$ and let the integer $m=2^{n}$. By Lemma 2.1, there exist an element $b_{n} \in A_{n} \otimes M_{m}$ and a $\sigma$-weakly continuous linear map $\Phi_{n}$ of $N$ into $M_{m}$ such that $\left\|b_{n}\right\| \leq 1,\left\|\Phi_{n}\right\| \leq n^{-1 / 8}$, $\Phi_{n} \mid A_{k} \equiv 0(k \neq n)$, and $\left\|\left(\Phi_{n} \otimes \mathrm{id}_{m}\right)\left(b_{n}\right)\right\| \geq 2^{-3 / 2} n^{3 / 8}$.

Now we put $\Phi=\bigoplus_{n=1}^{\infty} \Phi_{n}$; then $\|\Phi\|=\sup \left\|\Phi_{n}\right\| \leq 1$. Hence $\Phi$ is a $\sigma$ weakly continuous bounded linear map $N$ into $\bigoplus_{n=1}^{\infty} M_{m}$. Since $M$ is not 
strictly finite of type I, we can embed $\bigoplus_{n=1}^{\infty} M_{m}$ into $M$ as a von Neumann subalgebra. Hence $\Phi$ is a $\sigma$-weakly continuous bounded linear map of $N$ into $M$.

If $\Phi$ is completely bounded, we have $\|\Phi\|_{c b} \geq\left\|\Phi \mid W^{*}(a)\right\|_{c b}$. For each $k \epsilon$ $\mathbb{N}$, it is easy to see that $\Phi \otimes \mathrm{id}_{k}=\bigoplus_{n=1}^{\infty} \Phi_{n} \otimes \mathrm{id}_{k}$, so $\left\|\Phi \otimes \mathrm{id}_{k}\right\|=\sup _{n}\left\|\Phi_{n} \otimes \mathrm{id}_{k}\right\|$. Since $\left\|\Phi_{n} \otimes \mathrm{id}_{m}\right\| \geq 2^{-3 / 2} n^{3 / 8}$ is assured by Lemma 2.1, we have

$$
\|\Phi\|_{c b} \geq\left\|\Phi \mid A_{n}\right\|_{c b} \geq 2^{-3 / 2} n^{3 / 8} \text { for each } n \in \mathbb{N} \text {. }
$$

Hence, $\Phi$ is not completely bounded.

Theorem 2.5. Let $M$ and $N$ be two von Neumann algebras. Then the following assertions are equivalent:

(1) $B\left[M_{*}, N_{*}\right]=C B\left[M_{*}, N_{*}\right]$

(2) Either $M$ is strictly finite of type I or $N$ is finite-dimensional.

Proof. (1) $\rightarrow(2)$ : It follows from the previous lemma.

$(2) \rightarrow(1)$ : It follows from Smith's result.

Corollary 2.6. Let $A$ and $B$ be $C^{*}$-algebras. Then $B\left[A^{*}, B^{*}\right]=B\left[A^{*}, B^{*}\right]$ if and only if either $A$ is subhomogeneous or $B$ is finite-dimensional.

\section{ACKNOWLEDGMENT}

The author would like to express his hearty thanks to Professor S. Watanabe for his many valuable suggestions and advice and to Professor T. Huruya for much valuable advice and encouragement.

\section{REFERENCES}

1. E. G. Effros and Z.-J. Ruan, On matricially normed spaces, preprint.

2. U. Haagerup, Injectivity and decomposition of completely bounded maps, Lecture Notes in Math., Springer-Verlag, Berlin and New York, vol. 1132, pp. 170-222.

3. T. Huruya and J. Tomiyama, Completely bounded maps of $C^{*}$-algebras, J. Operator Theory 10 (1983), 141-152.

4. T. Ito, Completely bounded maps and a certain cross norm, Math. Japon. 32 (1987), 239249.

5. R. I. Loebl, Constructive linear maps on $C^{*}$-algebras, Michigan Math. J. 22 (1975), 361366.

6. __ Hahn decomposition for linear maps, Pacific J. Math. 65 (1976), 119-133.

7. T. Ogasawara, Finite dimentionality of certain Banach algebras, J. Sci. Hiroshima Univ. Ser. A 17 (1954), 359-364.

8. Z.-J. Ruan, Subspaces of $C^{*}$-algebras, J. Funct. Anal. 76 (1988), 217-230.

9. R. R. Smith, Completely bounded maps between $C^{*}$-algebras, J. London Math. Soc. (2) 27 (1983), 157-166.

10. T. Takasaki and J. Tomiyama, Stinespring type theorem for various tupes of completely positive maps associated to operator algebras, Math. Japon. 27 (1982), 129-139.

11. M. Takesaki, Theory of operator algebras I, Springer-Verlag, New York, Heidelberg, and Berlin, 1979. 
12. J. Tomiyama, Recent development of the theory of completely bounded maps between $C^{*}$. algebras, Publ. Res. Inst. Math. Sci. 19 (1983), 1283-1303.

13. S.-K. Tsui, Decomposition of linear maps, Trans. Amer. Math. Soc. 230 (1977), 87-112.

14. G. Wittstock, Ein operatorwertiger Hahn-Banach Satz, J. Funct. Anal. 40 (1981), 129-150.

Department of Mathematics, Tokyo Metropolitan University, Fukasawa, SetagayaKU 158, TOKYO, JAPAN 